CS Elizaveta Khachaturyan (ed.) Italiano e norvegese: studi di lingua e di cultura, Oslo Studies in Language 10(1), 2018. 119-141. (ISSN 1890-9639)

http://www. journals.uio.no/osla

\title{
IL LESSICO MUSICALE NEI MODI DI DIRE IN ITALIANO E IN NORVEGESE
}

\author{
MARGRETHE FØRRE NARDOCCI \\ Università di Oslo
}

ABS TRACT

This study describes Italian idiomatic expressions with musical lexis compared to Norwegian idioms from the same semantic field. Lexical and semantic criteria are used for the analysis. The corpus created for this purpose contains 157 expressions collected from different lexicographic sources. Several correspondences have been observed between Norwegian and Italian idioms: for example, string instruments are often used for expression describing moods, whereas wind instruments often have a negative connotation. At the same time, Italian idioms contain many key concepts that are often missing in Norwegian culture; for example, terms used to describe the liturgy and prayer (e.g., antifona 'antiphon', messa 'mass', gloria 'glory', vespro 'vespers', and litania 'litany'). Another interesting observation concerns the particular use of some verbs in Italian idioms: for example, suonare can signify 'to beat', and cantare come un canarino means 'to betray a secret'.

INTRODUZIONE

Questo articolo tratta dei modi di dire italiani che si sono formati a partire dal linguaggio musicale, un campo semantico dove la lingua italiana gode di una posizione di prestigio per essere considerata "la lingua della musica". L'argomento è poco studiato, nonostante l'importante influsso della musica nella diffusione degli italianismi nel mondo. Serianni afferma: "Si può dire che la lingua italiana faccia tutt'uno con la diffusione della musica occidentale" (2011: 227), facendo riferimento all'uso internazionale dei nomi di moltissimi strumenti musicali (pianoforte, viola, flauto), alle indicazioni agogiche come allegro, piano (usate anche nelle lingue come finnico, islandese, lituano, turco, georgiano, coreano, malese, indonesiano, giapponese, ecc.), e soprattutto, all'italiano come lingua del melodramma. Dato il prestigio della lingua italiana come lingua della musica, sarà quindi interessante in primo luogo esaminare come il campo semantico del lessico musicale si manifesta nella lingua italiana di tutti i giorni, e, in secondo luogo, paragonare due culture e lingue diverse: l'italiano ed il norvegese.

Il presente studio è basato su un vasto repertorio di espressioni idiomatiche italiane (157 unità raccolte dall'autore, tratte da diversi dizionari e repertori di 
modi di dire, in cui uno o più costituenti dell'espressione derivano dal campo semantico musicale). Per poter svolgere un'analisi contrastiva, abbiamo trovato, dove possibile, gli equivalenti norvegesi delle espressioni italiane e abbiamo effettuato il confronto basandoci sui criteri lessicali e semantici. L'obiettivo dell'analisi era quello di individuare convergenze e divergenze nei modi di dire delle due lingue a livello lessicale e semantico. Partendo dal presupposto che la musica è un fenomeno culturale universale, l'intento era quello di indagare su come l'esperienza e la storia musicale si sia manifestata nelle due lingue. ${ }^{1}$ Quali elementi musicali sono altrettanto importanti per le due culture e quali elementi sono tipici solo della cultura italiana? Quali sono gli elementi semantici in comune tra i modi di dire delle due lingue e quali sono le differenze? Sono le domande alle quali abbiamo cercato di rispondere.

\section{[1] APPROCCIO TEORICO}

\section{[1.1] Che cosa è un'espressione idiomatica}

Le espressioni idiomatiche non costituiscono una classe omogenea all'interno del linguaggio figurato di una lingua (Cacciari 1993: 27). Ogni espressione ha delle caratteristiche proprie che la differenziano dalle altre, così che la definizione e la classificazione delle espressioni idiomatiche diventa un compito piuttosto difficile. Nella classe di espressioni idiomatiche rientrano espressioni di origine diversa con le caratteristiche sintattiche, semantiche e pragmatiche molto varie. Nel corso degli ultimi anni sono stati condotti numerosi studi sull'argomento (Cacciari 1993, Casadei 1995, Gibbs 1993, Nunberg, Sag \& Wasow 1994) che però non hanno portato ad una definizione accettata da tutti i linguisti. Inoltre tutte le espressioni possono essere classificate a seconda della funzione sintattica che svolgono nella frase: verbale, nominale, aggettivale e avverbiale (Faloppa 2011).

Nelle definizioni proposte negli anni ‘ 50 -'70 (sotto l'influenza della grammatica generativa) la non-composizionalità insieme con la polilessicalità sono considerate le caratteristiche principali delle espressioni idiomatiche:

The essential feature of an idiom is that its full meaning...is not a compositional function of the meaning of the idiom's elementary grammatical parts.

(Katz \& Postal 1963: 275)

Questa definizione di Katz \& Postal può essere considerata la definizione "standard" di espressione idiomatica, mentre le definizioni più recenti sottolineano in genere due tratti: (i) le espressioni idiomatiche sono espressioni che contengono almeno due costituenti, (ii) il significato delle espressioni idiomatiche non è

[1] Questo argomento non è mai stato trattato prima. A quanto ci risulta, gli unici studi sul lessico musicale nelle espressioni idiomatiche italiane sono i lavori di Camugli-Gallardo, dove viene presentata un'analisi contrastiva tra italiano e francese $(1996,2003)$. 
deducibile dalla somma dei costituenti nell'espressione. La non-composizionalità di un'espressione significa che il suo significato non è distribuito tra le parti che la compongono. Per esempio, il significato 'morire' non può essere dedotto dalle parti che compongono l'espressione andare a far pelle di tamburo, oppure 'dire molto male di qualcuno, bestemmiare' non può essere ricavato dall'espressione tirar giù a campane doppie. Di conseguenza le espressioni idiomatiche risultano "bloccate" per quanto riguarda le modifiche che implicano l'autonomia semantica delle componenti interessate. Un'espressione come tirare le cuoia ${ }^{2}$ (morire) non ammette, senza perdere il senso idiomatico, né sostituzione di componenti (*tirare la pelle), né operazioni come modificazione del determinante (*Mario ha tirato delle cuoia), passivizzazione (*Le cuoia sono state tirate da Mario) o relativizzazione (*Le cuoia che Mario ha tirato erano vecchie).

Tuttavia, negli anni recenti, i linguisti (Nunberg, Sag \& Wasow 1994) e gli psicologi (Cacciari 1993, Gibbs 1993) hanno insistito sul fatto che la non-composizionalità come tratto definitorio per le espressioni idiomatiche non è sufficiente. Hanno rilevato che tante delle modifiche prima definite impossibili per un'espressione idiomatica sono invece attestate e giudicate accettabili dai parlanti. Casadei (1995), per esempio, analizza le espressioni togliersi un GROSSO peso dalla coscienza e prendere TRE piccioni con una fava, dove le modifiche effettuate sembrano rafforzare la lettura idiomatica anziché indebolirla o distruggerla. Questi esempi attestano la presenza e l'attivazione dei valori semantici dei singoli componenti dell'espressione idiomatica. Il problema, secondo Gibbs (1993) consiste nel fatto che le definizioni tradizionali si sono basate su un numero limitato di espressioni idiomatiche, solamente quelle più opache come per esempio kick the bucket ('morire'). Allo stesso tempo bisogna notare che alcune espressioni idiomatiche sono semanticamente trasparenti, come per esempio: dirigere l'orchestra ('essere a capo di un gruppo').

Così è diventato comune ritenere le caratteristiche elencate sopra non come assolute, ma come graduali. Quindi un'espressione può essere più o meno composizionale, più o meno fissa, più o meno opaca ecc. Infatti, Nunberg, Sag \& Wasow (1994) criticano tutti i tentativi di definire la nozione di espressione idiomatica in maniera troppo rigida e sostengono che la definizione di espressione idiomatica può variare a seconda dello scopo dello studio:

Idioms are not after all a linguistically natural kind, in the sense of being candidates for a category of universal grammar, and for theoretical purposes, the category can be defined in different ways for different purposes.

(Nunberg, Sag \& Wasow 1994: 494)

Siccome l'obiettivo del presente articolo è quello di studiare e analizzare i modi di dire che contengono il lessico musicale e di indagare su come l'esperienza e

[2] L'esempio è di Casadei 1996: 4 
la storia musicale si sia manifestata nella lingua, abbiamo scelto di raccogliere le espressioni idiomatiche, dando peso alla loro convenzionalità e al loro riferimento alla musica. Durante la costruzione del corpus abbiamo scoperto che poche sono le espressioni che veramente si possono definire soltanto in base alle caratteristiche già discusse di Katz \& Postal (1963): polilessicalità e non-composizionalità. Esempi come andare a far pelle di tamburo ('morire'), battere la solfa ('ripetere fino alla noia') o cantare la solfa a qualcuno ('rimproverare qd'), che sono completamente opache, idiomatiche, fisse e non-composizionali, sono rari. Si è scelto quindi di operare con una definizione ampia di espressione idiomatica che consenta di includere tanti tipi di espressioni.

Così, le espressioni idiomatiche raccolte per questo studio, hanno le seguenti caratteristiche:

- sono unità polilessicali con un significato convenzionale;

- hanno diversi gradi di composizionalità: da completamente non composizionali (nessuna componente partecipa al significato globale dell'espressione) (p.es. andare a far pelle di tamburo) a parzialmente composizionali (almeno uno dei costituenti mantiene il suo significato letterale) capire l'antifona, vibrare come una corda di violino, una sinfonia di colori, una litania di lamentele);

- hanno diversi gradi di trasparenza. Alcune sono del tutto opache, come andare a far pelle di tamburo o andare come i pifferi di montagna (si suppone che il parlante non conosca la motivazione etimologica dell'espressione), mentre le altre mostrano un grado più alto di trasparenza, come essere giù/su di tono, dare il la, essere sempre la solita canzone, toccare una corda sensibile;

- hanno diversi gradi di fissità sintattica: alcune non ammettono nessun tipo di modificazione mentre le altre ammettono modificazioni di vari tipi, come varianti di citazione (essere sempre la solita musica/solfa/ritornello, battere/insistere sempre sullo stesso tasto), passivizzazione (il tono è stato dato), inserzione di avverbi (essere/sembrare una corda un po' stonata), riformulazioni (sentire la campana di qcn. invece di sentire tutte (e due) le campane) (esempio di Casadei 1996).

Per costituire il corpus, abbiamo fatto ricorso a diversi dizionari e raccolte di modi di dire/espressioni idiomatiche: i dizionari Grande Dizionario Hoepli Italiano (Aldo Gabrielli 2011) e Italiensk blå ordbok (2002); le fonti lessicografiche disponibili in rete di Sabatini \& Coletti n.d. e della Treccani; i repertori dei modi di dire di Carollo (2008), Di Natale/ Zacchei (1996), Pittàno (2009), Quartu/ Rossi (2012), Sorge (2011); e il corpus di espressioni idiomatiche pubblicato da Casadei (1996) e Camugli-Gallardo (1996). Per trovare i modi di dire norvegesi abbiamo usato, oltre all'Italiensk blå ordbok, i libri di Erichsen $(1996,2011)$ e Vannebo $(2013)$ e anche 
la versione elettronica di Bokmålsordboka. L'uso incrociato di repertori specifici e dizionari generali è stato necessario in quanto manca una fonte completa di espressioni idiomatiche o modi di dire, sia per l'italiano che per il norvegese. Nemmeno per le espressioni idiomatiche contenenti lessico musicale esiste alcun tipo di raccolta.

Il criterio principale per scegliere un'espressione è stata la presenza di terminologia musicale, vale a dire che uno o più costituenti dell'espressione fanno parte del lessico musicale.

\section{[1.2] Espressioni idiomatiche - concetti chiave di una cultura}

I modi di dire sono portatori linguistici di costumi, tradizioni e storia e come tali sono spesso particolari per una lingua. Secondo Beccaria (2002: 196), la lingua è conservatrice per definizione. Così l'italiano dell'età della globalizzazione sembra ancora legato alla vita dei campi: le espressioni come cercare l'ago nel pagliaio o l'ultima ruota del carro sono ancora perfettamente comprensibili, benché i pagliai e i carri siano ormai ricordi lontani. Allo stesso modo, anche se la lira è stata ormai sostituita dall'euro, continuiamo ad usare le espressioni essere senza una lira, non guadagnare una lira e non essere senza un euro, non guadagnare un euro (tutti gli esempi sono tratti da Beccaria 2002: 196). Il ricorso ai modi di dire riflette quindi le esperienze che la gente ha vissuto in modo così intenso da imprimersi nella memoria collettiva, e rivela qualcosa di importante di noi stessi e della nostra storia. Kövecses (2010: 234) parla dell'importanza di concetti chiave (key concepts) in una cultura e in una comunità linguistica. Questi possono riguardare il sistema di valori, credenze popolari, ma possono anche riguardare concetti legati alla vita quotidiana. ${ }^{3}$ Anche i costumi particolari di una comunità linguistica possono dare origine alle espressioni idiomatiche specifiche di quella lingua: come è stato mostrato, per esempio, da alcuni studi sullo spagnolo dove la tradizione della festa nazionale - la corrida - ha dato origine a un gran numero di espressioni idiomatiche (per esempio Valero-Garcés 1997). Così l'analisi lessicale e semantica di un'espressione idiomatica può illustrare come la storia e la cultura del popolo si manifestano nella lingua di tutti i giorni.

L'Italia e la Norvegia hanno diversi elementi in comune nelle espressioni idiomatiche essendo due paesi che appartengono a quello che Piiranien chiama "the common European cultural heritage of classical and Christian provenance" (Piirainen 2012:517). Con questo termine Piirainen si riferisce alle tradizioni culturali che includono non solo l'antichità, il Cristianesimo, la letteratura biblica e Medie-

[3] Kövecses (2010) si riferisce a uno studio contrastivo tra inglese e francese condotto da Frank Boers e Murielle Demecheleer nel 1997 e nel 2001, dove gli autori sostengono che i concetti di HAT e SHIP sono più produttivi nelle espressioni idiomatiche metaforiche in inglese rispetto al francese, mentre $\mathrm{i}$ concetti di SLEEVE e FOOD sono più produttivi nelle espressioni idiomatiche francesi rispetto all'inglese. Secondo Boers e Demecheleer il motivo di questo sta nel fatto che i primi due concetti sono più salienti per i parlanti dell'inglese, mentre gli ultimi due sono più salienti per i parlanti del francese. 
vale latina, il Rinascimento, l'Umanesimo e l'Illuminismo, ma anche le opere della letteratura mondiale di vasta espansione. Secondo (Piirainen 2012: 48), ci sono alcuni fattori importanti per la diffusione delle espressioni idiomatiche, tra i quali si può citare la motivazione basata sull'intertestualità, che troviamo, per esempio, nelle espressioni to dance to someone's tune, ${ }^{4}$ ballare al suono di qualcuno, å danse etter ens pipe ${ }^{5}$ e to sing the same (old) song/tune. ${ }^{6}$ Queste espressioni sono esempi di quello che Piirainen definisce "widespread idiom": sono i modi di dire che esistono in tante lingue europee e che mostrano la stessa struttura lessicale e lo stesso significato. Piirainen (2012: 48) afferma che un criterio importante per la diffusione di un'espressione in tante lingue è la trasparenza semantica, vale a dire il grado di facilità con cui il significato idiomatico può essere ricavato solo in base alle parole che appaiono nell'espressione. Come vediamo dagli esempi riportati di seguito, le espressioni equivalenti in norvegese e in italiano sono caratterizzate da un alto livello di trasparenza:

(1) a. essere musica per le orecchie di qualcuno

b. å være musikk i noens ører

(2) a. essere/ sembrare un disco rotto

b. å være/ ha hakk i plata

(3) a. essere teso come una corda di violino

b. å være spent som en fiolinstreng

Secondo Piirainen (ibid.), le espressioni opache, quelle in cui è difficile capire il legame tra significato letterale e significato idiomatico, non avrebbero alcuna possibilità di diffondersi in tante lingue.

Allo stesso tempo, l'Italia e la Norvegia hanno una storia musicale abbastanza diversa. La musica italiana ha avuto un posto centrale nella vita musicale europea sin dal Medioevo, specialmente per lo sviluppo della tradizione operistica. L'Italia è spesso considerata la culla del melodramma e, di conseguenza, la lingua italiana viene definita nel mondo la "lingua del melodramma" (Serianni 2011). La

[4] La fonte principale di questa espressione è la favola di Esopo "Il pescatore flautista" che racconta la storia del pescatore che suona il flauto sperando che i pesci si precipitino da lui spontaneamente. Non avendo fortuna, smette di suonare e getta invece la rete in acqua, catturando questa volta molti pesci. La morale della favola è che i pesci non ballavano quando il pescatore suonava, ma solo quando erano costretti a farlo.

[5] To dance to someone's tune (Piirainen 2012: 447), ballare al suono (di qcn) (Casadei 1996: 215), å danse etter ens pipe (Bokmålsordboka).

[6] L'espressione si trova in opere antiche ed è menzionata anche negli Adagia (1505-1536) di Erasmo da Rotterdam (Piirainen 2016: 45): to sing the same (old) song/tune (Piirainen 2016: 44), è sempre la stessa canzone (Piirainen 2016: 46), samme gamle visa (Bokmålsordboka). 
storia musicale norvegese si basa invece in grande misura sulle tradizioni folcloristiche. Questo fattore potrebbe essere all'origine della scelta di parole diverse per le espressioni idiomatiche che troviamo nelle due lingue, come si vedrà nella sezione successiva.

[2] ANALISI DELLE ESPRESSIONI RACCOLTE

[2.1] Analisi lessicale

Le espressioni raccolte e analizzate possono essere suddivise in tre gruppi, a seconda degli elementi lessicali presenti nell'espressione:

(i) la musica intesa come "arte di combinare tra loro e di strutturare i suoni in un insieme armonico e unitario" (Dizionario Hoepli);

(ii) gli strumenti e le loro parti;

(iii) gli esecutori e l'esecuzione musicale.

Come vedremo, la terminologia usata nelle espressioni coincide in gran parte nelle due lingue. Analizziamo in modo più dettagliato ogni gruppo.

(i) Fanno parte del primo gruppo le espressioni dove l'elemento linguistico musicale si riferisce alla composizione musicale oppure ai suoi elementi. Si tratta di gruppi di parole che denotano diversi aspetti della musica in generale: ${ }^{7}$

- l'arte dell'organizzazione di suoni a livello generale: musica - musikk;

- il suono emesso e la sua caratteristica: registro, suono - lyd, tono - tone, unisono - enstemming / unisont;

- i segni con cui si rappresentano i suoni della musica nella notazione tradizionale: solfa, la, chiave; battuta - takt / opptakt, nota-noter;

- il supporto tecnico per la riproduzione sonora della musica o delle note: disco - plate, sordina - sordin; diapason;

- diversi generi di una composizione musicale: concerto, melodramma, toccata e fuga; canzone - sang / vise, sinfonia - symfoni, valzer - valsen;

- le parti di una composizione: preludio; ritornello - refereng, leitmotiv ledemotiv;

- vari componenti della liturgia cattolica: antifona, gloria, litania, messa, vespro;

- tecniche della composizione musicale: contrappunto, bordone.

[7] Come vediamo negli esempi che seguono non tutte le parole sono presenti nelle espressioni norvegesi. 
(ii) Nel secondo gruppo rientrano le espressioni contenenti parole che denotano gli strumenti e le parti degli strumenti. Possiamo dividere gli strumenti (i quali rappresentano spesso anche l'esecutore) nei seguenti gruppi:

- gli archi: cassa di risonanza-resonansbaum, corda-streng, sordina-sordin, violino - fele / fiolin;

- i legni: piffero ${ }^{8}$ - fløyte / pipe;

- gli ottoni: corno - basun (å utbasunere noe); tromba, trombone;

- le percussioni: campana, cimbali, tamburo - stortromme;

- strumenti a tastiera: organo, tasto.

(iii) Il terzo e ultimo gruppo è costituito dalle espressioni in cui l'elemento musicale si riferisce agli esecutori e all'atto di eseguire una composizione musicale: cantare - synge, coro - kor, orchestra - orkester, pianista - pianisten, primadonna - primadonna, suonare - spille; suonata, ${ }^{9}$ suonato.

Come possiamo vedere, c'è un alto livello di corrispondenza per quanto riguarda i lessemi musicali utilizzati nelle espressioni italiane e norvegesi. Nonostante queste similitudini, possiamo individuare differenze che dipendono dalla cultura e dalla storia di una specifica comunità linguistica. Alcuni termini sono assenti nelle espressioni norvegesi, come per esempio, i termini legati al solfeggio, come 'solfa' e 'la', ed i termini propri della preghiera e della liturgia, come 'antifona', 'messa', 'gloria', 'vespro' e 'litania'.

Per quanto riguarda l'assenza dei termini legati al solfeggio, ${ }^{10}$ il motivo potrebbe stare nel semplice fatto che il solfeggio come sistema di lettura musicale non è usato nei paesi nordici, dove le note sono indicate da lettere dell'alfabeto. Di conseguenza tali termini non sono tanto presenti nella realtà dei parlanti norvegesi e non fanno quindi parte di quelli che Kövecses (2010: 234) chiama concetti-chiave. L'importanza dei concetti-chiave si riflette anche nell'uso di termini propri della preghiera e della liturgia, concetti legati all'ambiente ecclesiastico. Lurati (2002) osserva come l'impatto del sacro e della componente cristia-

[8] Secondo il dizionario di Sabatini \& Coletti (consultato on-line http://dizionari.corriere.it/ dizionario_italiano/P/piffero.shtml) il piffero è uno "strumento a fiato simile al flauto diritto nella forma e all'oboe nel tono".

[9] Cfr. gli esempi di Sabatini \& Coletti: suonata di clacson - prendersi una bella suonata (bastonatura, grave sconfitta).

[10] Il solfeggio è il sistema di lettura musicale che consiste nel denominare le note musicali per mezzo di monosillabi (do, re, mi, fa, sol, la, si), ideato dal compositore italiano Guido d'Arezzo e risalente al XI secolo. Come disciplina, la pratica del solfeggio mira a sviluppare l'orecchio musicale, cioè la capacità di identificare le note, gli intervalli, le melodie e il ritmo, parte fondamentale dell'insegnamento della musica. Nei paesi nordici, al contrario, come anche in Inghilterra e in Germania, si è preferito invece mantenere fede al principio antichissimo di denominare i suoni con le lettere dell'alfabeto (C, D, E, F, G, A, H) (Pozzoli 1936). 
na nel discorso quotidiano italiano sia spesso maggiore di quanto si creda, come dimostra l'alto numero di espressioni che si rifanno ad una matrice religiosa:

Parecchi di quei modi di dire che giorno dopo giorno adoperiamo anche a proposito di cose lontane dal mondo spirituale si rifanno, in una maniera o l'altra all'esperienza religiosa, alla pastorazione, alle pratiche liturgiche.

(Lurati 2002: 153)

\section{E ancora:}

Certe espressioni quanto mai "laiche" e distanti dalla religione, si rivelano, a un'analisi sistematica, riflessi di componenti religiose, ciò nell'uso fraseologico di ogni giorno.

(Lurati 2002: 153)

Nella sua analisi Lurati fa notare che l'esistenza delle espressioni metaforiche ci permette di capire come venivano vissuti certi momenti della liturgia: finire in gloria ('finire bene'), cantare il vespro a qualcuno ('sgridare duramente'), una litania $d i+N$ ('cose noiose, lunghe, indeterminabili').

\section{[2.2] Analisi semantica}

Questa sezione presenta la classificazione semantica delle espressioni in base al loro significato idiomatico. Guardando le espressioni del corpus, emergono alcuni temi o significati ricorrenti che ritroviamo nelle due lingue. Possiamo constatare che, anche se gli esempi sono più numerosi in italiano che in norvegese, i significati e le connotazioni delle espressioni nei rispettivi domini semantici sembrano corrispondere nelle due lingue. Abbiamo individuato tre domini semantici principali: 'interazione e rapporti sociali', 'emozioni' e 'valutazione'. Per ogni dominio semantico, individuato in base alle espressioni italiane, troveremo almeno un esempio in norvegese che esprime lo stesso significato con le stesse connotazioni.

\section{Interazione e rapporti sociali}

Le espressioni che appartengono al dominio dell'interazione o dei rapporti sociali sono quelle che esprimono il modo attraverso il quale più persone interagiscono tra di loro. Questo dominio include un'immagine della musica come metafora della comunicazione e l'insieme musicale come metafora di un gruppo di persone.

Le espressioni nella tabella 1 richiamano l'aspetto collettivo tramite l'immagine del gruppo musicale come il coro o l'orchestra: 
TABELLA 1

\begin{tabular}{lll}
\hline Espressione italiana & $\begin{array}{l}\text { Definizione } \\
\text { dell'espressione italiana }\end{array}$ & $\begin{array}{l}\text { Espressione } \\
\text { norvegese }\end{array}$ \\
\hline Fare coro a qualcuno & Approvarne le opinioni & \\
\hline Tutti in coro & All'unanimità & Et kor av N (protester) \\
\hline $\begin{array}{l}\text { Un coro di N (lamentele, } \\
\text { proteste, lodi, fischi) }\end{array}$ & $\begin{array}{l}\text { Insieme di lamentele, } \\
\text { proteste, lodi fischi, } \\
\text { emessi da un gruppo } \\
\text { di persone concordemente }\end{array}$ \\
\hline Unirsi al coro & $\begin{array}{l}\text { Sostenere la stessa opinione } \\
\text { degli altri }\end{array}$ \\
\hline $\begin{array}{l}\text { Fare da contrappunto } \\
\text { a qcn }\end{array}$ & $\begin{array}{l}\text { Accompagnare, spalleggiare } \\
\text { con discorsi o azioni }\end{array}$ \\
\hline Tenere bordone a qcn & $\begin{array}{l}\text { Accompagnare, spalleggiare, } \\
\text { specialmente in qualcosa } \\
\text { disonesto }\end{array}$ \\
\hline
\end{tabular}

Vediamo che il repertorio di queste espressioni sembra più vasto in italiano. Per l'espressione fare coro a qualcuno non esiste un equivalente norvegese, mentre per le espressioni unirsi al coro e uscire/ levarsi/ staccarsi dal coro, dove la parola coro può essere interpretata come 'l'esempio da seguire', troviamo le espressioni norvegesi å følge flokken e å skille seg ut fra flokken ${ }^{11}$ (lett.: "seguire il branco" e "staccarsi dal branco") che esistono anche in italiano.

Alcune espressioni esprimono anche la modalità della comunicazione tramite la metaforizzazione dei rapporti tra persone come rapporti musicali (Casadei 1996: 373). Quando c'è l'armonia musicale, l'interazione è caratterizzata da un contenuto e/o un registro linguistico adatto alla situazione e da un'intesa tra le parti. Di conseguenza le espressioni riflettono l'aspetto positivo dell'interazione, come vediamo nella tabella 2:

Vediamo che in entrambe le lingue, la parola tono (in norvegese tone) rappresenta l'armonia musicale e quindi l'idea di "accordo" o "intesa" tra due parti. Anche l'idea di mettere d'accordo le persone nella stessa maniera in cui si accordano gli strumenti esiste in entrambe le lingue. In italiano viene usato il verbo "accordare" nell'espressione accordare gli strumenti ('mettere d'accordo persone, eliminare attriti'). In norvegese la stessa idea viene espressa attraverso la parola samstemme

[11] Gli esempi sono presi dal Bokmålsordboka 


\begin{tabular}{lll}
\hline Espressione italiana & $\begin{array}{l}\text { Definizione } \\
\text { dell'espressione italiana }\end{array}$ & Espressione norvegese \\
\hline Essere in sintonia & Capirsi, andare d'accordo & \\
\hline $\begin{array}{l}\text { Stare/rimanere/restare } \\
\text { in tono }\end{array}$ & $\begin{array}{l}\text { Non uscire dai termini del } \\
\text { discorso, parlare } \\
\text { appropriatamente }\end{array}$ & \\
\hline Trovare il tono giusto & $\begin{array}{l}\text { Trovare il modo migliore } \\
\text { per esprimere qualcosa }\end{array}$ & $\AA$ treffe riktig tone \\
\hline & Capirsi, andare d'accordo & A finne tonen \\
\hline & Buone maniere & Takt og tone \\
\hline
\end{tabular}

('essere in accordo/ armonia') e le espressioni samstemme i noe ('accordarsi su qualcosa') o samstemmende med noe ('essere in consonanza con qualcosa'). ${ }^{12}$ Inoltre, nel linguaggio giornalistico viene spesso usato il verbo harmonisere (armonizzare) che trasmette un'idea simile: De nordiske land harmoniserer sin handelspolitikk. ${ }^{13}$

Allo stesso tempo, la disarmonia musicale riflette un'interazione caratterizzata da attriti o da comportamenti "fuori luogo": essere fuori tono, uscire di tono ('stonare; non essere in sintonia con un ruolo o con un ambiente'), essere una no$t a /$ corda stonata ('essere un elemento non adatto, fuori luogo'). In norvegese esiste un'espressione å opptre umusikalsk che esprime la stessa idea di comportamenti fuori luogo: Hamsun-selskapet opptrer umusikalsk. ${ }^{14}$

All'interno del dominio dell'interazione troviamo anche le espressioni che esprimono il significato dei ruoli o del rango sociale, sempre tramite la metafora del gruppo musicale come gruppo di persone. La musica rappresenta un insieme armonico e la buona riuscita dell'interazione presuppone l'accordo preliminare degli strumenti, a partire dalla nota di referenza. Camugli-Gallardo (1996: 32), nel suo studio contrastivo sulle espressioni metaforiche fisse in italiano e francese, osserva come il 'tono' diventa il segno metonimico dell'esempio da seguire, una sorta di norma sociale: dare il tono (fare da esempio o da guida a un gruppo). Come vediamo, la stessa osservazione vale anche per il norvegese: $a$ slå an tonen, å vore toneangivende.

Le espressioni della tabella 3 hanno in comune l'idea di 'controllo della situa-

[12] Gli esempi sono presi dal Italiensk blå ordbok (2002: 312)

[13] L'esempio è preso dal Bokmålsordboka.

[14] L'esempio è preso da una trasmissione presentata su NRK: https://www.nrk.no/nordland/ krangler-om-hamsun-seminar-1.7941192 
zione':

TABELLA 3

\begin{tabular}{lll}
\hline Espressione italiana & $\begin{array}{l}\text { Definizione } \\
\text { dell'espressione italiana }\end{array}$ & $\begin{array}{l}\text { Espressione } \\
\text { norvegese }\end{array}$ \\
\hline Battere la solfa & Dirigere, comandare & \\
\hline Dirigere l'orchestra & $\begin{array}{l}\text { Essere a capo di un grup- } \\
\text { po }\end{array}$ & A svinge taktstokken \\
\hline Dare il diapason & $\begin{array}{l}\text { Dare il tono ad una con- } \\
\text { versazione o manifesta- } \\
\text { zione }\end{array}$ \\
\hline Dare il la & Dare inizio \\
\hline Dare il tono & $\begin{array}{l}\text { Fare da esempio o da } \\
\text { guida a un gruppo }\end{array}$ & $\begin{array}{l}\text { toneangivende } \\
\text { ton }\end{array}$ \\
\hline essere il primo violino & $\begin{array}{l}\text { Svolgere il ruolo più im- } \\
\text { portante, fare il protago- } \\
\text { nista }\end{array}$ \\
\hline
\end{tabular}

In queste espressioni l'orchestra è la metafora della piramide sociale. I ruoli importanti dell'orchestra come "il direttore" e "il primo violino", rappresentano i ruoli importanti nella vita sociale. Troviamo anche le espressioni per parlare delle persone sottomesse oppure appartenenti al basso rango sociale: ballare al suono di qualcuno / å danse etter noens pipe ('essere sottoposto alla volontà di qualcuno'), essere/fare il violino di spalla / å spille annen fiolin ('essere l'aiutante fedele di qualcuno, essere all'ombra del protagonista'). Le espressioni dare il tono e essere il primo violino fanno tutte e due parte di quel gruppo di espressioni che Piirainen chiama widespread idiom - WI (Piirainen 2016: 42-43), cioè espressioni che esistono in tante lingue europee e anche in lingue più distanti. È interessante osservare che per l'italiano non si trova un equivalente per l'espressione å spille første fiolin (suonare il primo violino). Secondo Piirainen l'espressione non si trova in nessuna delle lingue romanze occidentali, nelle quali esistono espressioni simili che sfruttano invece un'altra immagine, quella di un/una cantante: fare la primadonna, spagnolo llevar la voz cantante, catalano portar la veu cantant (Piirainen 2016: 42). Secondo la studiosa, la mancanza di equivalenti di una WI in una lingua può essere dovuta a vari fattori, tra i quali il fatto che esiste già un'espressione con lo stesso significato, sintatticamente simile ma lessicalmente diverso: "The most common cause is two or more 'competing' idioms, often idioms of a similar morpho-syntactic structure which are lexically different and therefore cannot be subsumed under a common 
widespread idiom" (Piirainen 2016: 673).

Un altro gruppo di modi di dire, sempre all'interno del dominio dell'interazione sociale', trasmette il significato di comunicazione, sia verbale che non-verbale. La comunicazione può avere caratteristiche positive (come negli esempi della tabella 4), ma anche caratteristiche negative (come nella tabella 5).

TABELLA 4

\begin{tabular}{lll}
\hline Espressione italiana & $\begin{array}{l}\text { Definizione } \\
\text { dell'espressione italiana }\end{array}$ & Espressione norvegese \\
\hline Cantare le lodi a qcn & $\begin{array}{l}\text { Lusingare o elogiare A lovsynge noen } \\
\text { qualcuno }\end{array}$ \\
\hline $\begin{array}{l}\text { Essere musica per le orec- } \\
\text { chie di qcn }\end{array}$ & $\begin{array}{l}\text { Detto quando senti dire A vcere musikk i noens ører } \\
\text { le cose che vorresti, per } \\
\text { esempio buone notizie }\end{array}$ \\
\hline $\begin{array}{l}\text { Fare una sviolinata/suona- } \\
\text { re il violino a qualcuno }\end{array}$ & $\begin{array}{l}\text { Lusingare o elogiare A rose noen i høye toner } \\
\text { qualcuno }\end{array}$ \\
\hline
\end{tabular}

In norvegese la parola "musikk" può addirittura rappresentare rapporti romantici, come nell'espressione å oppstå søt musikk (detto quando si crea una situazione romantica) oppure indicare qualcosa che richiede grande sforzo o impegno, come nell'espressione for full musikk ('con tutte le forze'). In italiano, invece, sembra che un numero maggiore di espressioni crei l'immagine negativa della comunicazione che in alcuni casi focalizza il contenuto negativo (come negli esempi 1-3 della tabella 5), mentre negli altri casi è la maniera di comunicare che ha delle caratteristiche negative (forte, inopportuno, clamoroso) (esempi 4-8):

Le caratteristiche negative della comunicazione sono spesso rappresentate dai fiati o dagli strumenti a percussione. Il suono intenso degli ottoni e delle campane può essere alla base del loro significato di messaggi 'fuori luogo' o 'inopportuni'. Come osserva Casadei (1996: 370) l'esprimersi in musica a volte introduce una maggiore enfasi rispetto all'esprimersi con suoni linguistici: mettere in musica (qcs) ('ripetere, chiarire per l'ennesima volta'), suonare/ suonarla in faccia/viso (qcs a qcn) ('dire chiaramente').

[15] L'espressione norvegese può però avere anche un altro significato, quello di "festeggiare o fare qualcosa con grande impeto, non badando a spese", come si può constatare nel seguente esempio: Slår på stortromma og gir flygel - Sparebanken Hedmark gir et konsertflygel og en gratiskonsert med den verdenskjente pianisten Leif Ove Andsnes i gave til det nye Kulturhuset $i$ Hamar. (https://www.nrk. no/ho/ slar-pa-stortromma-og-gir-flygel-1.10999021). In questo caso e con questo significato, le due espressioni sono solo apparentemente equivalenti.

[16] Il verbo spifferare con il primo significato 'riferire, ridire senza riserbo' deriva dal sostantivo piffero (dizionario Treccani). 
TABELLA 5

\begin{tabular}{ll}
\hline Espressione italiana & $\begin{array}{l}\text { Definizione } \\
\text { dell'espressione italiana }\end{array}$ \\
\hline 1. Cantarele proprie ragioni & $\begin{array}{l}\text { Dichiarare apertamente A synge ut sin mening } \\
\text { una posizione, anche ne- } \\
\text { gativa, nei confronti di } \\
\text { qualcuno }\end{array}$ \\
\hline $\begin{array}{l}\text { 2. Cantarne quattro a qual- } \\
\text { cuno }\end{array}$ & $\begin{array}{l}\text { Rimproverare, anche in- } \\
\text { sultare }\end{array}$ \\
\hline $\begin{array}{l}\text { 3. Tirar giù a campane dop- } \\
\text { pie }\end{array}$ & Bestemmiare molto \\
\hline $\begin{array}{l}\text { 4. Battere il tamburo/la } \\
\text { grancassa }\end{array}$ & $\begin{array}{l}\text { Fare propaganda chias- A slå påstortromma } \\
\text { sosa, cercare di attirare } \\
\text { attenzione }\end{array}$ \\
\hline $\begin{array}{l}\text { 5. Cantare (come un canari- } \\
\text { no) }\end{array}$ & $\begin{array}{l}\text { Confessare, tradire un se- } \\
\text { greto }\end{array}$ \\
\hline 6. Dare fiato alle trombe & $\begin{array}{l}\text { Annunciare } \\
\text { morosamente } \\
\text { qualcosa }\end{array}$ \\
\hline 7. Mettere & in \\
canzone/musica qcn & $\begin{array}{l}\text { Canzonare, metter in ri- } \\
\text { dicolo }\end{array}$ \\
\hline 8. Spifferare ${ }^{16}$ una notizia & Divulgare notizie segrete \\
\hline &
\end{tabular}


In italiano troviamo un gruppo particolare di espressioni che trasmettono il significato di comunicazione negativa. In questo caso la comunicazione equivale alla violenza fisica: dare una suonata a qualcuno ('imbrogliare qualcuno, bastonare qualcuno'), suonarle (di santa ragione) a qcn ('picchiare, malmenare').

Il verbo suonare e il suo derivato suonata trasmettono il significato di 'picchiare'. Può essere legato all'immagine degli strumenti a percussione, che appunto suonano quando percossi, colpiti, agitati o sfregati dalle mani del suonatore, o mediante appositi strumenti come bacchette o battenti. Nell'espressione italiana andare come i pifferi di montagna che andarono a suonare e furono suonati (che si riferisce a coloro che vanno a provocare e invece ne hanno la peggio) la polisemia del verbo suonare permette un gioco di parole relativo al ritorno imbarazzante dei pifferi dopo una partenza gloriosa (Camugli-Gallardo 1996).

È interessante osservare che in italiano il verbo suonare è spesso usato per esprimere il significato di 'sgridare' o 'picchiare qualcuno': una proiezione metaforica che non troviamo per la parola spille, l'equivalente norvegese di suonare. Anche il verbo cantare può voler dire 'tradire un segreto', significato che non esiste per il verbo synge in norvegese.

\section{Emozioni}

Nel dominio delle emozioni o dei sentimenti troviamo le espressioni che trasmettono uno stato d'animo, come, per esempio, allegria, tristezza o tensione. Gli elementi musicali più ricorrenti in questo dominio sono la parola tono e le parole legate agli strumenti ad arco (tabella 6).

TABELLA 6

\begin{tabular}{lll}
\hline Espressione italiana & $\begin{array}{l}\text { Definizione } \\
\text { dell'espressione italiana }\end{array}$ \\
\hline Essere giù di tono/di corda & Sentirsi deboli, abbattuti & Å vcere nedstemt \\
\hline Essere su di tono & $\begin{array}{l}\text { Sentirsi nel piano delle } \\
\text { forze }\end{array}$ \\
\hline Sentirsi in tono & $\begin{array}{l}\text { Sensazione oppstemt } \\
\text { generale }\end{array}$ \\
\hline
\end{tabular}

L'immagine trasmessa dalle espressioni riportate è quella dell'intonazione degli strumenti, che può essere "giusta" ('in tono'), troppo alta ('su') o troppo bassa ('giù'), e che rispecchia rispettivamente il sentirsi 'in equilibrio', 'felice' o 'triste'. Possiamo dire che queste espressioni sono correlate con la dimensione spaziale su-giù e le esperienze fisico-percettive legate ad essa: la posizione a capo chino si associa generalmente all'idea di tristezza e depressione (Lakoff \& Johnson 1980: 
34), la posizione a testa alta invece con uno stato emotivo positivo. ${ }^{17}$ Troviamo, inoltre, la variante che sfrutta la parola corda, una parte degli strumenti ad arco, in essere giù di corda.

Anche in altre espressioni gli archi trasmettono spesso il significato di uno stato d'animo: ad esempio, la 'tensione' in italiano e la 'felicità' in norvegese (tabella 7).

TABELLA 7

\begin{tabular}{lll}
\hline Espressione italiana & $\begin{array}{l}\text { Definizione } \\
\text { dell'espressione italiana }\end{array}$ & Espressione norvegese \\
\hline $\begin{array}{l}\text { Vibrare come una corda di } \\
\text { violino }\end{array}$ & $\begin{array}{l}\text { Avere grande sensibilità } \\
\text { fisica o psichica }\end{array}$ & Akjelve som et aspeløv \\
\hline $\begin{array}{l}\text { Essere teso come una corda } \\
\text { di violino }\end{array}$ & $\begin{array}{l}\text { Essere in uno stato di } \\
\text { grande tensione o nervo- } \\
\text { sismo }\end{array}$ & streng \\
\hline & Essere molto felice & A vcere glad som ei fele \\
\hline
\end{tabular}

Nelle espressioni italiane è usata la parola corda in associazione alle forme verbali vibrare e teso, caratteristiche della corda e del suono degli strumenti a cor$\mathrm{da}$, che danno rilievo all'accezione di 'essere in uno stato di grande tensione'. Dal punto di vista sintattico, queste espressioni hanno la forma di una comparazione e sono introdotte dalla parola 'come': fare come qualcosa. Secondo Piirainen (2008: 214), i concetti-chiave di una cultura sono particolarmente evidenti nelle similitudini, dove gli aspetti culturali si possono manifestare al livello del dominio-origine tramite le parole che denotano oggetti specifici di una cultura. L'espressione vibrare come una corda di violino trova il suo equivalente nell'espressione norvegese å skjelve som et aspeløv (lett.: 'tremare come una foglia di pioppo'), mentre l'espressione russare come un trombone può essere tradotta con å snorke som et sagbruk (lett.: 'russare come una segheria'). Queste espressioni sono equivalenti dal punto di vista semantico-strutturale, cioè esprimono lo stesso significato attraverso la stessa struttura sintattica, ma sfruttano domini di origine diversi. In norvegese il concetto della sensibilità è reso tramite l'immagine di una foglia di pioppo e il rumore assordante del russare tramite l'immagine di una segheria. La presenza di termini che hanno a che fare con gli alberi può essere legata al fatto che la foresta fin dai tempi antichi è stata un elemento molto importante per la Norvegia e per la sua base industriale, fornendo cibo, carburante, utensili, materiali per fare le case ecc.

[17] Questo sistema di relazioni sistematiche tra espressioni è stato elaborato nella teoria cognitiva della metafora da Lakoff \& Johnson (1980). 
Le altre espressioni contenenti la parola corda rimandano al significato di 'grande sensibilità' da parte dell'interlocutore, come per esempio toccare la corda giusta ('usare la tattica giusta per ottenere ciò che uno vuole') che trova il suo equivalente nell'espressione norvegese å spille på de riktige strengene. Un altro esempio è toccare una corda sensibile ('affrontare un argomento delicato'), equivalente all'espressione norvegese å berøre/spille på ømme strenger.

Nel paragrafo precedente si è visto come i fiati e gli strumenti a percussione nelle espressioni idiomatiche spesso descrivono una comunicazione inopportuna $o$ in qualche maniera negativa. Da questo punto di vista è interessante l'espressione andare/essere in cimbali ('manifestare grande allegria, specialmente dopo aver bevuto') dove i cimbali trasmettono la sensazione di 'grande allegria'. L'espressione sembra però mantenere la connotazione negativa legata agli strumenti a percussione, poiché l'allegria in questa espressione idiomatica è legata all'effetto di ebbrezza, si tratta cioè dell'allegria che si manifesta dopo aver bevuto. Gli strumenti ad arco invece, come abbiamo visto in questo paragrafo, spesso rappresentano la sfera dei sentimenti.

\section{Valutazione}

Siccome le espressioni idiomatiche sono spesso usate per sottolineare o attenuare il messaggio comunicativo, il loro uso non è neutro, ma trasmette l'atteggiamento positivo o negativo del locutore. Il dominio della valutazione riguarda le espressioni in cui il parlante vuole esprimere il suo parere valutativo su una situazione o su una persona. La valutazione può essere positiva o negativa. Si tratta di una valutazione positiva quando l'esito o il risultato di una faccenda viene visto in termini di 'successo': cantare vittoria ('rallegrarsi di un successo'), finire in gloria ('finire bene'). Bisogna osservare che le espressioni norvegesi che esprimono lo stesso significato sono fuori dal dominio della musica: per esempio å ikke selge skinnet før bjørnen er skutt ('non vendere la pelle dell'orso prima di averlo ucciso', che può essere definito come 'non cantare vittoria prima del tempo'), oppure å komme i havn / å dra i land ('andare in porto', nel senso di portare qualcosa al termine/ finire bene).

Una valutazione positiva può anche essere espressa tramite l'immagine di un "suono gradevole". In questo caso il contenuto di un messaggio o il comportamento di una persona è paragonato al suono piacevole della musica, come nell'espressione essere musica per le orecchie di qualcuno (usata quando si ricevono commenti o notizie positive), in norvegese å vcere musikk i noens ører, oppure nell'espressione essere tutta un'altra musica ('essere un discorso o una situazione del tutto diversi'), significato che si ritrova nell'espressione norvegese å vcere andre takter/ nye toner.

D'altra parte esiste un numero ancor maggiore di espressioni che trasmettono una valutazione negativa legata al carattere di una persona, ad una situazione $o$ al risultato negativo di una faccenda (tabella 8): 


\begin{tabular}{|c|c|c|}
\hline Espressione italiana & $\begin{array}{l}\text { Definizione } \\
\text { dell'espressione italiana }\end{array}$ & $\begin{array}{l}\text { Espressione } \\
\text { norvegese }\end{array}$ \\
\hline $\begin{array}{l}\text { Cambiare } i \text { suonatori ma } \\
\text { non la musica }\end{array}$ & $\begin{array}{l}\text { Cambiare una situazione solo } \\
\text { in apparenza, in senso negativo }\end{array}$ & \\
\hline $\begin{array}{l}\text { Essere come cantare ai sor- } \\
\text { di }\end{array}$ & $\begin{array}{l}\text { Parlare a persone che non } \\
\text { ascoltano }\end{array}$ & \\
\hline $\begin{array}{l}\text { Essere stonato come una } \\
\text { campana }\end{array}$ & Essere molto stonato & \\
\hline Essere trombato & $\begin{array}{l}\text { Essere bocciato alle elezioni, } \\
\text { essere estromesso }\end{array}$ & \\
\hline Essere una tromba & $\begin{array}{l}\text { Essere una persona pettegola, } \\
\text { intrigante, maldicente }\end{array}$ & \\
\hline Essere un vecchio trombone & $\begin{array}{l}\text { Essere un chiacchierone, pom- } \\
\text { poso, ciarlatano }\end{array}$ & \\
\hline $\begin{array}{l}\text { Non importare un } \\
\text { corno/piffero }\end{array}$ & Non importare niente & \\
\hline Non valere un corno & Non valere niente & \\
\hline \multirow[t]{2}{*}{$\begin{array}{l}\text { Tant'è suonare un corno } \\
\text { che un violino }\end{array}$} & $\begin{array}{l}\text { Prodigarsi inutilmente per una } \\
\text { persona non in grado di capire } \\
\text { quanto si fa per lei; capire poco } \\
\text { delle sfumature }\end{array}$ & \\
\hline & $\begin{array}{l}\text { Detto di qualcosa che si svol- } \\
\text { ge con difficoltà o in maniera } \\
\text { caotica, disordinata }\end{array}$ & Slinger i valsen \\
\hline
\end{tabular}


Nelle espressioni essere trombato/una tromba/un vecchio trombone, non valere un corno e non importare un piffero/un corno appare di nuovo la connotazione negativa dei fiati, già discussa sopra. Negli esempi riportati in questo paragrafo vediamo come la caratteristica fisica dei fiati, la loro forma cava/vuota, si presta ai rifiuti (un corno! non m'importa un piffero!).

In non valere un corno il significato negativo di 'non valere nulla' può inoltre essere legato alla forma e alle caratteristiche dello strumento, essendo nella versione antica uno strumento semplice, senza i cilindri del corno moderno francese, e che di conseguenza poteva emettere solo un numero limitato di suoni. Un'altra osservazione interessante legata alla parola 'corno' è fatta da Camugli-Gallardo (1996: 40), che fa notare come il fonema iniziale [k] seguito da una vocale aperta [o] produce un suono eclatante che contribuisce alla forza espressiva dell'espressione. La combinazione dell'occlusiva iniziale seguita da una vocale aperta non è riservata al campo lessicale della musica, ma la si trova anche in altre interiezioni italiane come cazzo, cavolo e caspita. Le espressioni con la parola corno possono, quindi, essere considerati eufemismi, cioè parole o frasi che sostituiscono altre parole o frasi con lo scopo di attenuare il carico espressivo di ciò che si vuole dire, perché ritenuto troppo offensivo o crudo (cfr. corno per cazzo).

Le espressioni tant'è suonare un corno che un violino e essere come cantare ai sordi sfruttano la figura retorica dell'antitesi che consiste nell'ottenere il rafforzamento di un concetto accostando due parole o concetti opposti: corno - violino, musica - sordi.

Basandosi sull'analisi del presente corpus, è possibile osservare uno squilibrio fra le espressioni con una connotazione positiva e quelle con una connotazione negativa. Il numero di espressioni che trasmettono una valutazione negativa è maggiore rispetto a quelle che trasmettono una valutazione positiva. Secondo Camugli-Gallardo (1996: 32), questo squilibrio può essere dovuto al fatto che i complimenti non hanno bisogno di essere nascosti, mentre i commenti critici devono essere smorzati, per esempio tramite un modo di dire, per non offendere o ferire i sentimenti dell'interlocutore. Per esempio, c'è una serie di espressioni che trasmette il significato di tedio dovuto alla ripetizione di un discorso o di una situazione. Alcune di queste espressioni sono caratterizzate da una struttura sintattica fissa che troviamo sia in norvegese che in italiano: $\mathrm{V}$ (essere/ripetere/vcere) - Avv (sempre/alltid) - art. Def - Agg (stesso/stessa/solito/solita/gamle) - N. Per esempio: essere sempre la solita/stessa antifona/canzone e ripetere sempre la solita solfa (ripetere sempre le stesse cose, fino ad annoiare. In norvegese troviamo, per esempio: $\stackrel{a}{a}$ allid vore den samme gamle visa.

Camugli-Gallardo (1996) osserva come in queste espressioni italiane vengono esaltati i rapporti fonetici tra parole tramite la ripetizione di suoni in parole successive: la [s] in sempre, solita, solfa. Possiamo trovare simili rapporti fonetici nell'espressione norvegese tramite la ripetizione di [am]: samme gamle nell'espressione 
å alltid voere den samme gamle visa.

La stessa idea della ripetizione si esprime anche attraverso il lessema che si riferisce al supporto meccanico della musica - il disco: il solito disco ('il solito discorso, la solita questione che si ripete'), essere/sembrare un disco rotto ('parlare sempre della stessa cosa, tornare sempre sullo stesso argomento'), equivalente all'espressione norvegese å vœre/ha hakk i plata.

Il tedio espresso in queste frasi dichiarative si trova anche in alcune espressioni imperative che esprimono il desiderio di cambiamento: Cambia disco! o Cambia musica! ('cambia discorso o argomento'). Di questa serie di espressioni si possono individuare alcuni equivalenti totali, ossia espressioni in cui c'è una corrispondenza semantico-strutturale nelle due lingue, per esempio: essere sempre la solita/stessa antifona/canzone - å alltid vore den samme gamle visa e essere/sembrare un disco rotto - å vore/ha hakk i plata.

\section{[3] CONCLUSIONE}

Le espressioni analizzate sopra sono state raggruppate secondo il loro contenuto lessicale e il significato semantico. Abbiamo visto nella classificazione lessicale che esiste un alto livello di corrispondenza tra i lessemi musicali utilizzati nelle espressioni italiane e norvegesi. La parola 'tono' è tra le più usate nelle espressioni idiomatiche, sia in norvegese che in italiano. Allo stesso tempo, alcuni termini sembrano non esistere nelle espressioni norvegesi, come per esempio la parola 'solfa' e i termini propri della preghiera e della liturgia, come 'antifona', 'messa', 'gloria', 'vespro' e 'litania'. Questa differenza può essere spiegata attraverso la teoria di Kövecses (2010) che propone di individuare i concetti-chiave (key concepts) di una cultura e di una comunità linguistica.

Nel confrontare le espressioni italiane con le equivalenti norvegesi abbiamo visto che, a livello semantico, i significati e le connotazioni delle espressioni corrispondono nelle due lingue. Nella classificazione semantica sono stati individuati diversi temi o significati ricorrenti nelle espressioni di entrambe le lingue: interazione e rapporti sociali, emozioni e valutazione. Abbiamo visto, per esempio, che le parole legate agli strumenti ad arco trasmettono spesso uno stato d'animo (essere giù di corda - å vcere nedstemt; essere su di corda - å vcere oppstemt), mentre le parole legate agli strumenti a fiato assumono piuttosto una connotazione negativa (dare fiato alle trombe - å utbasunere noe; non valere un corno; non importare un piffero). Il tedio dovuto alla ripetizione di un discorso o di una situazione è espresso in maniera simile nelle due lingue, tramite espressioni che condividono la stessa struttura sintattica: essere sempre la stessa/la solita canzone/solfa/antifona - å alltid vore den samme gamle visa; essere/sembrare un disco rotto - å voere/ha hakk i plata. Abbiamo anche osservato la distribuzione diversa di alcune parole molto comuni nelle due lingue, come 'cantare' e 'suonare'. La parola 'suonare' assume in tante espressioni idiomatiche il significato di 'picchiare', una proiezione meta- 
forica che non esiste per la parola 'spille' in norvegese. Analogamente, la parola 'cantare' in cantare come un canarino esprime un significato che non esiste per la parola 'synge' in norvegese, quello di 'tradire un segreto'.

\section{RIFERIMENTI BIBLIOGRAFICI}

Beccaria, G. L. 2002. Italiano, oggi: l'antico, il nuovo. Cuadernos de Filologia Italiana 9. 191-203.

Cacciari, C. 1991. La metafora: Da evento del linguaggio a struttura del pensiero.

In C. Cacciari (cur.), Teorie della metafora. L'aquisizione, la comprensione e l'uso del linguaggio figurato, 1-27. Milano: Raffaello Cortina Editore.

Cacciari, C. 1993. The Place of Idioms in a Literal and Metaphorical world. In C. Cacciari \& P. Tabossi (cur.), Idioms: Processing, Structure, and Interpretation, 2756. New Jersey: Psychology Press.

Camugli-Gallardo, C. 1996. Un regard différent sur la réalité extra-linguistique? Étude d'un domaine métaphorique italien. Transalpina. Regards croisés. Caen: Presses Universitaires de Caen, 29-52.

Camugli-Gallardo, C. 2003. Qu'est-ce que tu chantes là? Syntaxe e lexique dans les expressions métaphorique figées. Cahiers de lexicologie 82(1). Paris: Honoré Champion Paris, 175-192.

Casadei, F. 1995. Flessibilità lessico-sintattica e produttività semantica delle espressioni idiomatiche: Un'indagine sull'italiano parlato. In F. Casadei, G. Fiorentino \& V. Samek-Lodovici (cur.), L'italiano che parliamo, 11-33. Santarcangelo: Fara Editore.

Casadei, F. 1996. Metafore ed espressioni idiomatiche. Uno studio semantico sull'italiano. Roma: Bulzoni editore.

Gibbs, R. W. 1993. Why Idioms Are Not Dead Metaphors. In C. Cacciari \& P. Tabossi (cur.), Idioms: Processing, Structure, and Interpretation, 57-78. New Jersey: Psychology Press.

Granger, S. \& M. Paquot. 2008. Disentangling the phraseological web. In S. Granger \& F. Meunier (cur.), Phraseology: An interdisciplinary perspective, 27-50. Amsterdam: John Benjamins Publishing Company.

Katz, J. J. \& P. N. Postal. 1963. Semantic interpretation of idioms and sentences containing them. Reasearch Laboratory of Electronics Quarterly Progress Report 70. M.I.T., 275-282.

Kövecses, Z. 2005. Metaphor in Culture. Universality and Variation. New York: Cambridge University Press.

Kövecses, Z. 2010. Metaphor. New York: Oxford University Press.

Lakoff, G. \& M. Johnson. 1980. Metaphors we live by. Chicago: University of Chicago Press.

Lurati, 0. 2002. Per Modo di dire... Storia della lingua e antropologia nelle locuzioni italiane ed europee. Bologna: CLUEB. 
Nunberg, G., I. Sag \& T. Wasow. 1994. Idioms. Language 70(3). 491-538.

Piirainen, E. 2008. Figurative phraseology and culture. In S. Granger \& F. Meunier (cur.), Phraseology: An interdisciplinary perspective, 207-228. Amsterdam: John Benjamins Publishing Company.

Piirainen, E. 2012. Widespread idioms in Europe and Beyond. Toward a Lexicon of Common Figurative Units. New York: Peter Lang Publishing.

Piirainen, E. 2016. Lexicon of Common Figurative Units. Widespread Idioms in Europe and Beyond. Volume II. In cooperation with József Attila Balázsi. New York: Peter Lang Publishing.

Serianni, Luca. 2011. L'italiano nel mondo. In Vittorio Coletti (cur.), L'italiano dalla nazione allo Stato, 227-231. Firenze: Le Lettere.

Sinclair, J. 2008. Preface. In. Phraseology: An interdisciplinary perspective. S Granger \& F. Meunier (cur.). Amsterdam: John Benjamins Publishing Company. Xv-xviii.

Valero-Garcés, C. 1997. Contrastive idiomatology. Equivalence and translatability of english and spanish idioms. Poznan Studies in Contrastive Linguistics 32.29-38.

\section{Dizionari}

Carollo, S. (cur.). 2008. La vera storia di 400 frasi celebri e modi di dire. Firenze: Giunti Demetra.

Di Natale, F. \& N. Zacchei (cur.). 1996. In bocca al lupo. Espressioni idiomatiche e modi di dire tipici della lingua italiana. Perugia: Guerra Edizioni.

Erichsen, G. M. (cur.). 1996. Fraseologismer fra norsk til tysk. Oslo: Cappelen Akademisk Forlag.

Erichsen, G. M. (cur.). 2011. Ord og uttrykk på fire språk. Oslo: Cappelen Damm Akademisk.

Gabrielli, A. (cur.). 2011. Grande Dizionario Hoepli Italiano. Milano: Ulrico Hoepli Editore.

Italiensk blå ordbok, italiensk - norsk/ norsk - italiensk. 2002. Oslo: Kunnskapsforlaget. Pittàno, G. (cur.). 2009. Frase fatta capo ha. Dizionario dei modi di dire, proverbi e locuzioni di italiano. Bologna: Zanichelli.

Quartu, M. \& E. Rossi (cur.). 2012. Dizionario dei modi di dire della lingua italiana. Milano: Hoepli Editore.

Sorge, P. (cur.). 2011. Dizionario dei modi di dire della lingua italiana. Origine e significato delle frasi idiomatiche e delle forme proverbiali rare e comuni. Roma: Newton Compton editori.

Vannebo, K. I. 2013. Prikken over i-en og andre uttrykk. Bruk - bakgrunn - betydning. Oslo: Cappelen Damm.

\section{Sitografia}

Faloppa, F. 2011. Modi di dire. http: //www . treccani.it/enciclopedia/modidi-dire_\%28Enciclopedia_dell\%27Italiano\%29/ (2 marzo, 2014). 
Kunnskapsforlaget (cur.). 2015. Norsk Riksmålsordbok. http : // www . ordnett . no (30 marzo, 2015).

Pozzoli, E. 1936. Solfeggio.http://www . treccani.it/enciclopedia/solfeggio_ (Enciclopedia_Italiana)/ (15 ottobre, 2014).

Sabatini \& Coletti. n.d. Il Sabatini Coletti Dizionario della Lingua Italiana. http : / / dizionari.corriere.it/dizionario_italiano/.

Universitet i Oslo (cur.). 2010. Bokmålsordboka. http: //www . nob-ordbok . uio . no (20 marzo, 2015). 